Proceedings of the Edinburgh Mathematical Society (2007) 50, 325-341 (C)

DOI:10.1017/S0013091505000738 Printed in the United Kingdom

\title{
THE GROUP OF COVERING AUTOMORPHISMS OF A QUASI-COHERENT SHEAF ON $\boldsymbol{P}^{1}(K)$
}

\author{
E. ENOCHS ${ }^{1}$, S. ESTRADA ${ }^{1,2}$, J. R. GARCÍA ROZAS ${ }^{3}$ AND L. OYONARTE ${ }^{3}$ \\ ${ }^{1}$ Department of Mathematics, University of Kentucky, \\ Lexington, KY 40506-0027, USA (enochs@ms.uky.edu) \\ ${ }^{2}$ Departamento de Matemática Aplicada, Universidad de Murcia, \\ Campus del Espinardo, Espinardo (Murcia) 30100, Spain (sestrada@ual.es) \\ ${ }^{3}$ Departamento de Álgebra y A. Matemático, Universidad de Almería, \\ Almería 04071, Spain (jrgrozas@ual.es; oyonarte@ual.es)
}

(Received 12 May 2005)

\begin{abstract}
CoGalois groups appear in a natural way in the study of covers. They generalize the wellknown group of covering automorphisms associated with universal covering spaces. Recently, it has been proved that each quasi-coherent sheaf over the projective line $\boldsymbol{P}^{1}(R)(R$ is a commutative ring) admits a flat cover and so we have the associated coGalois group of the cover. In general the problem of computing coGalois groups is difficult. We study a wide class of quasi-coherent sheaves whose associated coGalois groups admit a very accurate description in terms of topological properties. This class includes finitely generated and cogenerated sheaves and therefore, in particular, vector bundles.
\end{abstract}

Keywords: quasi-coherent sheaf; flat cover; coGalois group

2000 Mathematics subject classification: Primary 18F20; 16G20; 20F38

Secondary 16B50; 14F45

\section{Introduction}

CoGalois groups were first defined in [7] for torsion-free covers of abelian groups. It was observed that these coGalois groups have a natural topology that is compatible with their group structure. Then, noting that coGalois groups are the categorical notion dual to that of an absolute Galois group of a field and that these groups with their natural topology are always compact, it was natural to ask about the compactness of these coGalois groups. In [7] it was shown that these groups are not always compact and necessary and sufficient conditions for their compactness were given.

In this paper we want to consider analogous questions in a different setting. We will consider torsion-free covers in the category of quasi-coherent sheaves on a scheme. We note that such a sheaf is 'locally a module' and so, if the scheme is the projective line $\boldsymbol{P}^{1}(k)$ (with $k$ a field), we will be considering modules over $k[x], k\left[x^{-1}\right]$ and $k\left[x, x^{-1}\right]$ (see $[\mathbf{1}]$ or $\S 2$, below, for an explanation of this point of view). We now give a brief outline of the contents of the paper. 
In $\S 2$ we explain how we view the category of quasi-coherent sheaves on a projective line. Then we recall the definition of covers and give some of the main tools used to prove their existence.

In $\S 3$ we give the main reduction result about computing coGalois groups of torsionfree covers over a Dedekind domain. Therefore, we show that every such coGalois group is isomorphic to one associated with a cotorsion module.

In $\S 4$ we study the topology on our coGalois groups and in particular consider when they have a certain local compactness.

Our main results are in $\S 5$. There we fully exploit the reduction result of $\S 3$ and give our locally compact version of the main result of [7, Theorem 5.2].

\section{Preliminaries}

Throughout the paper, all rings considered will be commutative with identity.

In the following we let $Q \operatorname{co}\left(\boldsymbol{P}^{1}(R)\right)$ or $Q \operatorname{co}\left(\boldsymbol{P}_{R}^{1}\right)$ denote the category of quasi-coherent sheaves over $\boldsymbol{P}^{1}(R)$ (where $R$ is any ring).

It is known (see, for example, $[\mathbf{1}, \mathbf{5}, \mathbf{8}, \mathbf{9}]$ ) that the structure sheaf of the projective line may be identified with the representation

$$
\mathcal{O} \equiv R[x] \hookrightarrow R\left[x, x^{-1}\right] \hookleftarrow R\left[x^{-1}\right]
$$

of the quiver $Q \equiv \bullet \rightarrow \bullet \leftarrow \bullet$. And in this case a quasi-coherent sheaf $\mathcal{L} \in Q \operatorname{co}\left(\boldsymbol{P}_{R}^{1}\right)$ is identified with a representation

$$
\mathcal{L} \equiv M \stackrel{f}{\rightarrow} P \stackrel{g}{\leftarrow} N
$$

of the quiver $Q$, where $M \in R[x]$-Mod, $N \in R\left[x^{-1}\right]$-Mod and $P \in R\left[x, x^{-1}\right]$-Mod and are such that $S^{-1} f$ and $T^{-1} g$ are isomorphisms of $R\left[x, x^{-1}\right]$-Mod (where $S=\left\{1, x, x^{2}, \ldots\right\}$ and $\left.T=\left\{1, x^{-1}, x^{-2}, \ldots\right\}\right)$. With this notation, a quasi-coherent sheaf $F_{1} \rightarrow F \leftarrow F_{2}$ is flat if and only if it is locally flat (that is, $F_{1}, F_{2}$ and $F$ are flat $R[x], R\left[x^{-1}\right]$ and $R\left[x, x^{-1}\right]$ modules, respectively). Analogously, if $R$ is Noetherian, a quasi-coherent sheaf $E_{1} \rightarrow E \leftarrow E_{2}$ is injective if and only if $E_{1}, E_{2}$ and $E$ are injective $R[x], R\left[x^{-1}\right]$ and $R\left[x, x^{-1}\right]$ modules, respectively (see $[\mathbf{9}]$ ). When $R=k$ (a field), since the class of flat (respectively, injective) $k[x], k\left[x^{-1}\right]$ and $k\left[x, x^{-1}\right]$ modules coincides with the class of torsion-free (respectively, divisible) modules, we find that the class of flat (respectively, injective) quasi-coherent sheaves on $Q c o\left(\boldsymbol{P}_{k}^{1}\right)$ coincides with the class of torsionfree (respectively, divisible) quasi-coherent sheaves.

We recall from [3] that, given a class $\mathcal{F}$ of objects in an abelian category, an $\mathcal{F}$-precover of an object $M$ is a morphism $\varphi: F \rightarrow M$ with $F \in \mathcal{F}$, such that for any other morphism $\varphi^{\prime}: F^{\prime} \rightarrow M$ with $F^{\prime} \in \mathcal{F}$ there exists $f: F^{\prime} \rightarrow F$ such that $\varphi \circ f=\varphi^{\prime}$. The $\mathcal{F}$-precover is said to be an $\mathcal{F}$-cover if every endomorphism $g: F \rightarrow F$ satisfying that $\varphi \circ g=\varphi$ is in fact an automorphism. Following [7], we will let $\operatorname{coGal}(\varphi)$ denote the group of automorphisms associated with the cover. In a dual manner we may define the notions of $\mathcal{F}$-(pre)envelopes and the corresponding Galois group of an envelope. 
Suppose that the category $\mathcal{C}$ has flat objects (so we are assuming that $\mathcal{C}$ is a category with a tensor product and the flat objects are those $F \in \mathrm{Ob}(\mathcal{C})$ such that $F \otimes \cdot$ is exact). If $\mathcal{F}$ is the class of all flat objects, we shall call an $\mathcal{F}$-(pre)cover a flat (pre)cover. The right orthogonal class of the class of flat objects, that is, the class

$$
\mathcal{F}^{\perp}=\left\{C \in \mathrm{Ob}(\mathcal{C}): \operatorname{Ext}^{1}(F, C)=0, \forall F \in \mathcal{F}\right\}
$$

is called the class of cotorsion objects. For an arbitrary class $\mathcal{F}$, the pair $\left(\mathcal{F}, \mathcal{F}^{\perp}\right)$ is said to be cogenerated by a set if there exists a subset $X \subseteq \mathcal{F}$ such that $X^{\perp}=\mathcal{F}^{\perp}$. A pair $\left(\mathcal{F}, \mathcal{F}^{\perp}\right)$ is a cotorsion theory if

$$
{ }^{\perp}\left(\mathcal{F}^{\perp}\right)=\left\{F \in \mathcal{F}: \operatorname{Ext}^{1}(F, C)=0, \forall C \in \mathcal{F}^{\perp}\right\}=\mathcal{F} .
$$

Cotorsion theories were first introduced in [14] for abelian groups. In [11, Corollary 4.1.10] it is shown that for a Grothendieck category $\mathcal{C}$, if the pair $\left(\mathcal{F}, \mathcal{F}^{\perp}\right)$ is cogenerated by a set, then it is a cotorsion pair whenever the class $\mathcal{F}$ is closed under direct sums, extensions and well-ordered direct limits, and a generator of $\mathcal{C}$ is in $\mathcal{F}$.

A cotorsion theory $\left(\mathcal{F}, \mathcal{F}^{\perp}\right)$ is said to be hereditary if for each exact sequence

$$
0 \rightarrow F \rightarrow F^{\prime} \rightarrow F^{\prime \prime} \rightarrow 0
$$

with $F^{\prime}, F^{\prime \prime} \in \mathcal{F}, F$ is also in $\mathcal{F}$.

Now, in [5, Corollary 4.2], it is proved that each object of $Q c o\left(\boldsymbol{P}_{R}^{1}\right)$ (with $R$ any ring) has a flat cover and a cotorsion envelope. This is done by showing that the pair $\left(\mathcal{F}, \mathcal{F}^{\perp}\right)$ composed by flat quasi-coherent sheaves and cotorsion quasi-coherent sheaves of $Q \operatorname{co}\left(\boldsymbol{P}_{R}^{1}\right)$ is cogenerated by a set. Moreover, by the comments above, since the family

$$
\mathcal{O}(n) \equiv R[x] \hookrightarrow R\left[x, x^{-1}\right] \stackrel{x^{n}}{\longleftarrow} R\left[x^{-1}\right], \quad n \in \mathbb{Z},
$$

is a family of (flat) generators for $Q \operatorname{co}\left(\boldsymbol{P}_{R}^{1}\right)$, the pair $\left(\mathcal{F}, \mathcal{F}^{\perp}\right)$ is a cotorsion theory. When we consider quasi-coherent sheaves over the projective line on a field $k$, the cotorsion theory is hereditary [9].

\section{General results}

The next two general results (Proposition 3.1 and Theorem 3.2) deal with a reduction of the problem of computing the coGalois group of a quasi-coherent sheaf.

Proposition 3.1. Let $(\mathcal{F}, \mathcal{C})$ be a cotorsion theory in a Grothendieck category $\mathcal{A}$ such that the generator of $\mathcal{A}$ is in $\mathcal{F}$. If

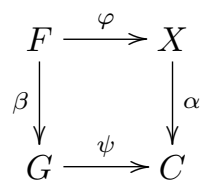

is a pull-back diagram where $\alpha$ is a $\mathcal{C}$-envelope and where $\psi$ is an $\mathcal{F}$-cover, then $\beta$ is a $\mathcal{C}$-envelope. If $(\mathcal{F}, \mathcal{C})$ is hereditary, then $\varphi$ is also an $\mathcal{F}$-cover. 
Proof. Since the diagram is a pull-back and $\alpha$ is a monomorphism, $\beta$ is also a monomorphism. By one of the Wakamatsu lemmas [16, Lemma 2.1.2] $\operatorname{coker}(\alpha) \in \mathcal{F}$, so $\operatorname{coker}(\beta) \cong \operatorname{coker}(\alpha) \in \mathcal{F}$. Another Wakamatsu lemma [16, Lemma 2.1.1] shows that $\operatorname{ker}(\psi) \in \mathcal{C}$. So $G \in \mathcal{C}$ and then $\beta$ is a $\mathcal{C}$-pre-envelope (in fact a special $\mathcal{C}$-pre-envelope, following [16, p. 29], that is, a monomorphism whose cokernel is in $\mathcal{F}$ ). Suppose that $t \beta=\beta$ for an endomorphism $t: G \rightarrow G$. We show that $t$ is an automorphism. We take the morphism $\psi t: G \rightarrow C$. Since the diagram is also a push-out, there will exist a morphism $k: C \rightarrow C$ such that $k \alpha=\alpha$ and $k \psi=\psi t$. Since $\alpha$ is an envelope, $k$ must be an automorphism, so we have two covers $k \psi, \psi: G \rightarrow C$, and therefore $t$ must be an automorphism.

Now we show that $\varphi$ is an $\mathcal{F}$-cover. If $(\mathcal{F}, \mathcal{C})$ is hereditary, then since $\operatorname{coker}(\beta), G \in \mathcal{F}$, $F \in \mathcal{F}$. We also have $\operatorname{ker}(\varphi) \cong \operatorname{ker}(\psi) \in \mathcal{C}$, so $\varphi$ is an $\mathcal{F}$-precover (in fact a special $\mathcal{F}$-precover). Suppose $d: F \rightarrow F$ is such that $\varphi d=\varphi$. We show that $d$ is an automorphism. Since $\beta: F \rightarrow G$ is an envelope, there will exist $t: G \rightarrow G$ such that $t \beta=\beta d$. If we consider the morphism $\psi t: G \rightarrow C$, we have

$$
\psi t \beta=\psi \beta d=\alpha \varphi d=\alpha \varphi,
$$

and, since the diagram is a push-out, we find $r: C \rightarrow C$ with $r \alpha=\alpha$ and $r \psi=\psi t$. But $\alpha$ is an envelope, so $r$ is an automorphism. So the previous reasoning shows that $t$ is also an automorphism. But $r: C \rightarrow C, t: G \rightarrow G$ (and id $: X \rightarrow X$ ) being automorphisms and using the pull-back property we conclude that $d$ is also an automorphism.

There exists a canonical morphism of groups between the coGalois groups involved in the square of Proposition 3.1. For if we take $f \in \operatorname{coGal}(\psi)$ we can consider the morphism $f \beta: F \rightarrow G$ and, since the diagram is a pull-back, there will exist a unique $h \in \operatorname{coGal}(\varphi)$ with $f \beta=\beta h$. It is straightforward to see that this is a morphism of groups. Moreover, one of the main results of [10] points out that the previous morphism is an epimorphism. We summarize the previous comments in the next theorem.

Theorem 3.2. Let $(\mathcal{F}, \mathcal{C})$ be a hereditary cotorsion theory in a Grothendieck category and let

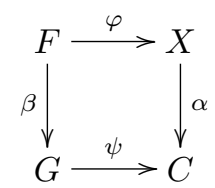

be a pull-back diagram where $\alpha$ is a $\mathcal{C}$-envelope and $\psi$ is an $\mathcal{F}$-cover. There is then a naturally defined surjective group homomorphism

$$
\operatorname{coGal}(\psi) \rightarrow \operatorname{coGal}(\varphi),
$$

where the kernel is in a bijective correspondence with $\operatorname{Hom}(\operatorname{coker}(\beta), \operatorname{ker}(\varphi))$.

Proof. The proof appears in $[\mathbf{1 0}$, Theorem 1.6]. 
From now on, $k$ will denote an algebraically closed field.

Let $\mathcal{M}$ be an element of $Q \operatorname{co}\left(\boldsymbol{P}_{k}^{1}\right)$, and let $\alpha: \mathcal{M} \rightarrow \mathcal{C}$ and $\psi: \mathcal{G} \rightarrow \mathcal{C}$ be a cotorsion envelope of $\mathcal{M}$ and a flat cover of $\mathcal{E}$, respectively. We consider the pull-back square of $\alpha$ and $\psi$

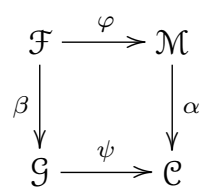

By Proposition $3.1 \varphi$ is a flat cover and $\beta$ is a cotorsion envelope, and thus Theorem 3.2 gives an epimorphism

$$
\operatorname{coGal}(\psi) \rightarrow \operatorname{coGal}(\varphi) \rightarrow 1
$$

(notice that the hereditary condition holds by the comments at the end of $\S 2$ ). It follows that, in order to study the coGalois group of a flat cover of an object, we should first consider cotorsion objects. Moreover, since the class of cotorsion objects is closed under extensions, the flat cover of a cotorsion module will also be a cotorsion, so we can use the results given in $[\mathbf{9}]$ concerning the structure of the flat and cotorsion quasi-coherent sheaves of $Q \operatorname{co}\left(\boldsymbol{P}_{k}^{1}\right)$ to compute the coGalois groups. After that we shall study the structure of the kernel of the previous epimorphism of groups. This will allow us to clarify the relation between the two coGalois groups.

In $\S 4$ we will relate the category $Q \operatorname{co}\left(\boldsymbol{P}_{R}^{1}\right)$ and the category of $R[x]$-modules (respectively, $R\left[x^{-1}\right]$-modules) for any $\operatorname{ring} R$. When $A$ is a Dedekind domain, the epimorphism (3.1) is in fact an isomorphism [10, Theorem 2.3]. So, putting together these two facts, we describe the coGalois group of a quasi-coherent sheaf and give a structure result (Theorem 5.3) for those quasi-coherent sheaves of $\boldsymbol{P}^{1}(k)$ having certain topological properties. The next theorem summarizes the relevant information from [10] that we shall use later.

Theorem 3.3. Consider the category of $R$-modules, with $R$ a Dedekind domain, $X$ an $R$-module and the pull-back



where $\alpha$ is a cotorsion envelope and $\psi$ a flat cover. Then the two coGalois groups $\operatorname{coGal}(\varphi)$ and $\operatorname{coGal}(\psi)$ are isomorphic.

We also notice that if $C$ is a cotorsion $R$-module (with $R$ still a Dedekind domain) and $C=V \oplus C^{\prime}$, where $V$ is the maximal torsion-free and divisible submodule of $C$, the coGalois groups of the torsion-free covers of $C^{\prime}$ and $C$ are isomorphic. For if $\varphi$ : $V \oplus F^{\prime} \rightarrow V \oplus C^{\prime}$ is the torsion-free cover of $C$, we conclude that $\operatorname{ker}(\varphi)=\operatorname{ker}\left(\varphi^{\prime}\right)$, where 
$\varphi^{\prime}: F^{\prime} \rightarrow C^{\prime}$ is the torsion-free cover of $C^{\prime}$, so

$$
\begin{aligned}
\operatorname{coGal}(F \rightarrow C) & \cong \operatorname{Hom}(F, \operatorname{ker}(\varphi)) \\
& \cong \operatorname{Hom}(F \oplus V, \operatorname{ker}(\varphi) \oplus 0) \operatorname{Hom}\left(F^{\prime}, \operatorname{ker}\left(\varphi^{\prime}\right)\right) \\
& \cong \operatorname{coGal}\left(F^{\prime} \rightarrow C^{\prime}\right)
\end{aligned}
$$

Therefore, we have proved that, to compute coGalois groups over a Dedekind domain, we can reduce them to cotorsion modules having no non-zero torsion-free and divisible submodules.

\section{Locally decomposable coGalois groups on $P^{1}(k)$}

Henceforth, $k$ will denote an algebraically closed and complete field, for example, the field of complex numbers or the $p$-adic completion of an algebraic closure of the field of $p$-adic numbers (see [13, $\S$ III.4 70]).

In order to study the coGalois group of a flat cover of a quasi-coherent sheaf $M \rightarrow P \leftarrow$ $N$ in $Q c o\left(\boldsymbol{P}_{R}^{1}\right)$ (with $R$ any ring), it is worthwhile to note that the problem can be reduced in a local way by considering the modules on the extremes. This is possible because there exists an adjoint pair of functors $(H, D)$ in this situation. The existence of such functors is proved in [9]. We now recall their definitions. The functor $H: Q \operatorname{co}\left(\boldsymbol{P}_{R}^{1}\right) \rightarrow R[x]$-Mod is defined by mapping a representation $\mathcal{F} \equiv A \rightarrow C \leftarrow B$, to the $R[x]$-module $A$, and for a morphism $(\alpha, \beta, \gamma)$ between two representations, $H(\alpha, \beta, \gamma)=\alpha$. The right adjoint functor of $H$ is the functor $D: R[x]-\operatorname{Mod} \rightarrow Q \operatorname{co}\left(\boldsymbol{P}_{R}^{1}\right)$ defined as follows: for any $L \in R[x]$-Mod, $D(L)$ is the representation $L \stackrel{i}{\rightarrow} S^{-1} L \stackrel{\text { id }}{\longleftarrow} S^{-1} L$ (where $S=\left\{1, x, x^{2}, \ldots\right\}$ and $i: L \rightarrow S^{-1} L$ is the natural morphism, induced by $\left.R[x] \rightarrow S^{-1} R[x]\right)$, and for an $R[x]$-morphism $\alpha: L \rightarrow M, D(\alpha)=\left(\alpha, S^{-1} \alpha, S^{-1} \alpha\right)$. Analogously, we may define the corresponding pair of adjoint functors $\left(H^{\prime}, D^{\prime}\right)$ between the categories $R\left[x^{-1}\right]$-Mod and $Q \operatorname{co}\left(\boldsymbol{P}_{R}^{1}\right)$. So we have the isomorphism

$$
\operatorname{Hom}_{Q c o\left(\boldsymbol{P}_{R}^{1}\right)}(D(A), D(M)) \cong \operatorname{Hom}_{R[x]}(A, M),
$$

for any $A, M \in R[x]$-Mod. We also have the following easy result.

Lemma 4.1. Let $M \rightarrow P \leftarrow N$ be a quasi-coherent sheaf in $Q \operatorname{co}\left(\boldsymbol{P}_{R}^{1}\right)$. If $\varphi: F \rightarrow M$ is the flat cover of $M$ (respectively, $\varphi^{\prime}: F^{\prime} \rightarrow N$ is the flat cover of $N$ ), then $D(\varphi)$ (respectively, $D^{\prime}\left(\varphi^{\prime}\right)$ ) is the flat cover of $D(M)$ (respectively, a flat cover of $D^{\prime}(N)$ ).

Proof. This is a particular case of [9, Corollary 3.2] by taking as $\mathcal{F}$ the class of all flat quasi-coherent sheaves and $\mathcal{F}_{1}, \mathcal{F}_{3}$ the class of flat $R[x]$-modules and $R\left[x^{-1}\right]$-modules respectively.

So in some sense the previous result allows us to reduce the problem of computing coGalois groups on $Q c o\left(\boldsymbol{P}_{R}^{1}\right)$ to computing them in $R[x]$-Mod. In the case when the ring $R$ is a Dedekind domain, it is known (see $[\mathbf{6}, \S 3]$ ) that, for a flat cover $\varphi: F \rightarrow D$ of a cotorsion $R$-module $D$, the corresponding coGalois group, $\operatorname{coGal}(\varphi)$, can be expressed as 
the product, indexed by all the non-zero primes of $R$, of coGalois groups of flat covers of localizations on each prime, that is,

$$
\operatorname{coGal}(\varphi) \cong \prod_{0 \neq p} \operatorname{coGal}\left(\varphi_{p}\right)
$$

where $\varphi_{p}$ is a flat cover of a cotorsion module over $R_{p}$, for all $0 \neq p \in \operatorname{Spec}(R)$. Therefore, to study the coGalois group of a flat cover (or equivalently in this situation a torsionfree cover) of a $k[x]$-module, we need only consider the flat cover of a cotorsion module $M \in k[x]_{p}$-Mod, with $0 \neq p \in \operatorname{Spec}(k[x])$.

Let us now define a topology on the coGalois group of the torsion-free cover of each cotorsion $k[x]_{p}$-module $M$. Let $\mathcal{F}$ be a class in an abelian category. If $\varphi: F \rightarrow M$ is an $\mathcal{F}$ cover with kernel $L$, there exists a canonical bijection between $\operatorname{coGal}(\varphi)$ and $\operatorname{Hom}(F, L)$, that is, morphisms $F \rightarrow F \in \operatorname{Hom}(F, F)$ with image lying in $L$ (see [7, $\S 4]$ ), that maps an element $f \in \operatorname{coGal}(\varphi)$ (so $\varphi \circ f=\varphi$ ) to $f-\operatorname{id}_{F}: F \rightarrow L$. We shall denote by ' $\square$ ' the group operation induced in $\operatorname{Hom}(F, L)$ by the usual composition of maps in $\operatorname{coGal}(\varphi)$, so, given $f^{*}, g^{*} \in \operatorname{Hom}(F, L)$ (that is, $\left.f=\operatorname{id}_{F}+f^{*}, g=\operatorname{id}_{F}+g^{*}\right), f^{*} \square g^{*}=f^{*}+g^{*}+f^{*} \circ g^{*}$. Now we take the category of $k[x]_{p}$-modules and the class $\mathcal{F}$ as the class of torsion-free modules. With the previous notation, if we define a topology on $T_{p}$ (the completion of a free $k[x]_{p}$-module with respect to the $p_{p}$-adic topology) for every $\left.p \in \operatorname{Spec}(k[x])\right)$, this will give a topology on $\operatorname{Hom}(F, L)$ and hence on the coGalois group (since $F, L$ are torsion-free and cotorsion $k[x]_{p}$-modules, by [4, Theorem], $L \cong U_{p}$ and $F \cong T_{p}$, with $U_{p}$, $T_{p}$ the completions of free $k[x]_{p}$-modules with respect to the $p_{p}$-adic topology). So we say $O \subseteq T_{p}$ to be open if $p^{n} T_{p} \subseteq O$ for some $n \geqslant 1$ and $\left(O / p^{n} T_{p}\right) \cap S$ is an open subset of $S$, for every finite-dimensional vector subspace $S$ (with the separated topology) of the $k$-vector space $T_{p} / p^{n} T_{p}$.

Finally, we notice that, since each open subset $O$ of $T_{p}$ under this topology is open in the $p$-adic topology, we may easily conclude from the argument of $[\mathbf{7}$, p. 239] that the topology is compatible with the group operation $\square$ and hence with the operation of the coGalois group.

Remark. The previous topology allows us to make more precise the statement of Theorem 3.2. So with the notation of that theorem we have a short exact sequence of topological groups

$$
1 \rightarrow \operatorname{Hom}(\operatorname{coker}(\beta), \operatorname{ker}(\varphi)) \rightarrow \operatorname{coGal}(\psi) \rightarrow \operatorname{coGal}(\varphi) \rightarrow 1 .
$$

Example 4.2. Consider the $k[x]$-module $k=k[x] /(x)$. The torsion-free cover is

$$
\varphi: k \llbracket x \rrbracket \rightarrow k, \quad s(x) \mapsto s(0) .
$$

The elements of the coGalois group $G$ are the series $1+a_{1} x+a_{2} x^{2}+\cdots \in k \llbracket x \rrbracket$, because

$$
\operatorname{Hom}(k \llbracket x \rrbracket, x k \llbracket x \rrbracket) \cong x \operatorname{Hom}(k \llbracket x \rrbracket, k \llbracket x \rrbracket) \cong x k \llbracket x \rrbracket .
$$

A subset $O \subseteq k \llbracket x \rrbracket$ will be open if $x^{n} k \llbracket x \rrbracket \subseteq O$ for some $n \geqslant 1$ and $O=U+x^{n} k \llbracket x \rrbracket$, where $U$ is an open subset of $k^{n}$ with the separated topology, so in this case the topology we define on $k \llbracket x \rrbracket$ is just the product topology $\left(k \llbracket x \rrbracket \cong k^{\mathbb{N}}\right)$. 
It is also worth noting that in the previous example the dimension as a $k \cong k[x] /(x)$ vector space of the quotient $k \llbracket x \rrbracket / x k \llbracket x \rrbracket$ is finite (equal to 1 ), so $G / G^{x}$ (where $G^{x}$ denotes the open subgroup of $G$ corresponding to $x \operatorname{Hom}(k \llbracket x \rrbracket, x k \llbracket x \rrbracket))$ is a locally compact topological vector space (see, for example, $[\mathbf{1 5}$, Chapter $1, \S 3.6]$ ). This justifies Definition 4.4.

Lemma 4.3. The subset $p \operatorname{Hom}(F, L) \subseteq \operatorname{Hom}(F, L), 0 \neq p \in \operatorname{Spec}(k[x])$, is an open normal subgroup of $\operatorname{Hom}(F, L)$ with the group operation induced by $\operatorname{coGal}(\varphi)$. Moreover, the quotient group

$$
\operatorname{Hom}(F, L) / p \operatorname{Hom}(F, L)
$$

is a vector space over the field $k[x] / p$.

Proof. Let us consider $f^{*}, g^{*} \in \operatorname{Hom}(F, L)$. Then $f^{*} \square g^{*}=f^{*}+g^{*}+f^{*} \circ g^{*}$. Since $L \subseteq p F$ (see [2, Theorem 2.1]), it follows that the group operation induced in the quotient $\operatorname{Hom}(F, L) / p \operatorname{Hom}(F, L)$ is merely the usual addition of maps, so it is a $k[x] / p$-vector space.

Definition 4.4. Let $G$ be the coGalois group of a torsion-free cover of a $k[x]_{p}$-module $(0 \neq p \in \operatorname{Spec}(k[x]))$, and let $G^{p}$ be the open subgroup of $G$ corresponding to the open subgroup $p \operatorname{Hom}(F, L)$. Then $G$ is said to be locally compact modulo $G^{p}$ if $G / G^{p}$ is a locally compact topological vector space over $k \cong k[x] / p$.

Remark. The previous definition is motivated by the situation in $\mathbb{Z}_{p}$. In this case it is shown in [11, Corollary 5.6.4] that the coGalois group $G$ is locally compact (equivalently compact) if, and only if, $G / G^{p}$ is finite. The finiteness is replaced in our case by finite dimensionality. But we cannot expect even local compactness for $G$ in our case (note that in Example $4.2 k \llbracket x \rrbracket$ is neither compact nor locally compact with the product topology).

We now give a complete classification of the cotorsion $k[x]$-modules having coGalois groups that are locally compact modulo $G^{p}$. By Theorem 3.3 and the comment after it, we need only to consider cotorsion $k[x]_{p}$-modules having no non-zero torsion-free and divisible submodules, with $p \in \operatorname{Spec}(k[x])$. We recall that an $R$-module $(R$ is a Dedekind domain) is said to be reduced if it has no non-zero divisible submodules.

Theorem 4.5. Let $C$ be a cotorsion $k[x]_{p}$-module $(0 \neq p \in \operatorname{Spec}(k[x]))$ with no nonzero torsion-free and divisible submodules, let $\varphi: F \rightarrow C$ be a flat cover of $C$ with kernel $L$, and let $G$ be its associated coGalois group. Then $G$ is locally compact modulo $G^{p}$ if, and only if,

$$
C \cong E(k[x] / p)^{m} \oplus \frac{k[x]}{p^{k_{1}}} \oplus \cdots \oplus \frac{k[x]}{p^{k_{s}}} \oplus \widehat{k[x]}_{p}^{n},
$$

where $E(\cdot)$ denotes the injective envelope.

\section{Proof.}

Case $1(\Rightarrow)$. A cotorsion $k[x]_{p}$-module as before has the form $C \cong E(k[x] / p)^{(X)} \oplus D$, where $D$ is a reduced and cotorsion $k[x]_{p}$-module (see $[\mathbf{1 2}, \S 54(\mathrm{I})]$ ). The flat cover will be $F=S^{-1} T \oplus U$ (with $S=k[x]-\{0\}$ ), where $T$ and $U$ are torsion-free and cotorsion $k[x]_{p}$-modules (so then are completions of free $k[x]_{p}$ modules with respect to the $p_{p}$-adic 
topology; see [4, Theorem]) and the kernel $L=T \oplus V$ (with $V$ a torsion-free and cotorsion $k[x]_{p}$-module as before), where

$$
0 \rightarrow V \rightarrow U \rightarrow D \rightarrow 0
$$

and

$$
0 \rightarrow T \rightarrow S^{-1} T \rightarrow E(k[x] / p)^{(X)} \rightarrow 0
$$

are the torsion-free covers of $D$ and $E(k[x] / p)^{(X)}$, respectively (the latter is proved in $[6$, Theorem 2.1]). By hypothesis, $\operatorname{Hom}(F, L) / p \operatorname{Hom}(F, L)$ is finite dimensional. But we have the isomorphisms

$$
\frac{\operatorname{Hom}(F, L)}{\operatorname{Hom}(F, p L)} \stackrel{(*)}{\cong} \operatorname{Hom}\left(F, \frac{L}{p L}\right) \cong \operatorname{Hom}\left(\frac{F}{p F}, \frac{L}{p L}\right),
$$

where the isomorphism ( $*$ ) follows because $0 \rightarrow p L \rightarrow L \rightarrow L / p L \rightarrow 0$ is a flat cover by [16, Proposition 4.1.6] and $p L$ is a cotorsion module by a Wakamatsu lemma [16, Lemma 2.1.1]. We deduce that $F / p F$ and $L / p L$ are finite-dimensional $k[x] / p$-vector spaces, and hence $T / p T, U / p U$ and $V / p V$ are also finite-dimensional $k[x] / p$-vector spaces. In the case $T / p T$ this says that $X$ must be finite (namely $|X|=m$ ). Suppose that $U=\widehat{k[x]}]_{p}^{n}$. By the structure theorem for modules over a principal ideal domain (in this case $\widehat{k[x]_{p}}{ }_{p}$ ) we have

$$
D \cong \frac{k[x]}{p^{k_{1}}} \oplus \cdots \oplus \frac{k[x]}{p^{k_{s}}} \oplus \widehat{k[x]}_{p}^{n-s}
$$

so $L=\widehat{k[x]}_{p}^{m} \oplus \widehat{k[x]}_{p}^{s}, F=S^{-1} \widehat{k[x]}_{p}^{m} \oplus \widehat{k[x]}_{p}^{n}$ and we conclude that $C$ is as stated.

Case $2(\Leftarrow)$. We must show that $\operatorname{dim}_{k} \operatorname{Hom}(F, L) / p \operatorname{Hom}(F, L)<\infty, k \cong k[x] / p$. But $\operatorname{Hom}(F, L) \cong \widehat{k[x]}_{p}^{r}$ for certain $r \in \mathbb{N}$ so

$$
\frac{\operatorname{Hom}(F, L)}{p \operatorname{Hom}(F, L)} \cong \frac{\widehat{k[x]}_{p}^{r}}{p \widehat{k[x]}_{p}^{r}} \cong\left(\frac{k[x]}{p}\right)^{r},
$$

which is obviously finite dimensional.

Remark. The previous theorem, together with Theorem 3.3, allows us to give an explicit description of the elements of the coGalois group locally compact modulo $G^{p}$, $0 \neq p \in \operatorname{Spec}(k[x])$, similar to that obtained in $[6, \S 4]$.

We apply Theorem 4.5 in order to find a family of quasi-coherent sheaves of $Q \operatorname{co}\left(\boldsymbol{P}_{k}^{1}\right)$ having coGalois groups that are locally compact modulo $G^{p}$.

Proposition 4.6. The coGalois group $G$ of the torsion-free cover of a quasi-coherent sheaf of the form

$$
D\left(E(k[x] / p)^{m}\right) \oplus D\left(k[x] / p^{k_{1}}\right) \oplus \cdots \oplus D\left(k[x] / p^{k_{s}}\right) \oplus D\left(\widehat{k[x]_{p}^{l}}\right)
$$


with $p \in \operatorname{Spec}(k[x])$, or

$$
D^{\prime}\left(E\left(k\left[x^{-1}\right] / q\right)^{b}\right) \oplus D^{\prime}\left(k\left[x^{-1}\right] / q^{t_{1}}\right) \oplus \cdots \oplus D^{\prime}\left(k\left[x^{-1}\right] / q^{t_{r}}\right) \oplus D^{\prime}\left(\widehat{k\left[x^{-1}\right]_{q}^{m}}\right)
$$

with $q \in \operatorname{Spec}\left(k\left[x^{-1}\right]\right)$, is locally compact modulo $G^{p}$ or modulo $G^{q}$, respectively.

Proof. By Lemma 4.1 (and comments preceding it), Theorem 4.5 and observing that

$$
\begin{aligned}
D\left(E(k[x] / p)^{m}\right) & \equiv E(k[x] / p)^{m} \rightarrow E(k[x] / p)^{m} \leftarrow E(k[x] / p)^{m}, \\
D\left(k[x] / p^{k}\right) & \equiv k[x] / p^{k} \rightarrow k[x] / p^{k} \leftarrow k[x] / p^{k}
\end{aligned}
$$

and

$$
D\left(\widehat{k[x]}_{p}^{l}\right) \equiv \widehat{k[x]}_{p}^{l} \rightarrow \widehat{k[x]}_{p}^{l} \leftarrow \widehat{k[x]}_{p}^{l}
$$

whenever $p \neq(x)$ and if $p=(x)$, we obtain

$$
\begin{aligned}
D\left(E(k[x] /(x))^{m}\right) & \equiv E(k[x] /(x))^{m} \rightarrow 0 \leftarrow 0, \\
D\left(k[x] /(x)^{k}\right) & \equiv k[x] /(x)^{k} \rightarrow 0 \leftarrow 0
\end{aligned}
$$

and

$$
D\left(\widehat{k[x]}_{(x)}^{l}\right) \equiv k \llbracket x \rrbracket^{l} \rightarrow k\left[x^{-1}, x \rrbracket^{l} \leftarrow k\left[x^{-1}, x \rrbracket^{l},\right.\right.
$$

where $k\left[x^{-1}, x \rrbracket\right.$ denotes the ring of formal power series in $x$ with a finite number of powers in $\left.x^{-1}\right)$. An analogous result holds for the functor $D^{\prime}$ with the corresponding $k\left[x^{-1}\right]$-modules.

\section{The main theorem and its consequences}

We shall now find families of quasi-coherent sheaves over $\boldsymbol{P}^{1}(k)$ whose coGalois groups decompose as the product of locally compact coGalois groups (modulo the respective subgroups). We do this by studying cotorsion envelopes. First we note the following remarkable fact.

Lemma 5.1. There are no non-zero torsion-free and cotorsion quasi-coherent sheaves over $\boldsymbol{P}^{1}(k), \mathcal{C} \equiv C_{1} \rightarrow C \leftarrow C_{2}$ with $C_{1}$ a reduced, torsion-free and cotorsion $k[x]_{(x)^{-}}$ module, and $C_{2}$ a reduced, torsion-free and cotorsion $k\left[x^{-1}\right]_{\left(x^{-1}\right)}$-module.

Proof. Suppose there exists such a non-zero representation. Then, by $[\mathbf{1 6}$, Theorem 4.1.15], $C_{1}$ (respectively, $C_{2}$ ) will be the completion of a free $k[x]_{(x)}$-module (respectively, the completion of a free $k\left[x^{-1}\right]_{\left(x^{-1}\right)}$-module), so, following the usual notation for such a completion, we denote $C_{1}$ by $U_{(x)}$ and $C_{2}$ by $V_{\left(x^{-1}\right)}$. Now, by [9, Theorem 4.7], $\mathcal{C}=D(A) \oplus D^{\prime}(B)$, where $A$ and $B$ are direct summands of $U_{(x)}$ and $V_{\left(x^{-1}\right)}$, respectively. Such direct summands are again of the form $M_{(x)}$ and $N_{\left(x^{-1}\right)}$ [16, Lemma 4.1.11], so we say that $C_{1}=M_{(x)} \oplus T^{-1} N_{\left(x^{-1}\right)}$. But $T^{-1} N_{\left(x^{-1}\right)}$ is a divisible $k\left[x, x^{-1}\right]$-module and hence a divisible $k[x]$-module, and, since $C_{1}$ is reduced, it must be equal to 0 . Now, $N_{\left(x^{-1}\right)}$ is torsion free, so it must be 0 . Analogously, we may deduce that $A$ is also 0 , which is a contradiction. 
Theorem 5.2. Let $\mathcal{N}$ be a quasi-coherent sheaf over $\boldsymbol{P}^{1}(k)$ and let $\varphi: \mathcal{G} \rightarrow C(\mathcal{M})$ be the torsion-free cover of the cotorsion envelope of $\mathcal{M}$. Then

$$
\operatorname{coGal}(\varphi) \cong \prod_{(x) \neq p \in \operatorname{Spec}(k[x])} \operatorname{coGal}\left(\varphi_{p}\right)
$$

Proof. Let $C(\mathcal{M}) \equiv C_{1} \rightarrow C \leftarrow C_{2}$. By an easy modification of the argument preceding $\S 4$, we may suppose that $C(\mathcal{M})$ does not contain a torsion-free and divisible quasi-coherent subsheaf. By [9, Lemma 5.1], $C_{1}$ and $C_{2}$ are cotorsion $k[x]$ and $k\left[x^{-1}\right]$ modules, respectively, so

$$
C_{1}=\bigoplus_{p \in \operatorname{Spec}(k[x])} E(k[x] / p)^{\left(X_{p}\right)} \oplus \prod_{(x) \neq p \in \operatorname{Spec}(k[x])} L_{p} \oplus L_{(x)},
$$

and

$$
C_{2}=\bigoplus_{q \in \operatorname{Spec}\left(k\left[x^{-1}\right]\right)} E\left(k\left[x^{-1}\right] / q\right)^{\left(Y_{q}\right)} \oplus \prod_{\left(x^{-1}\right) \neq q \in \operatorname{Spec}\left(k\left[x^{-1}\right]\right)} U_{q} \oplus U_{\left(x^{-1}\right)},
$$

with $L_{p}$ (respectively, $U_{q}$ ) cotorsion and reduced $k[x]$-modules (respectively, $k\left[x^{-1}\right]$ modules), $0 \neq p \in \operatorname{Spec}(k[x])$ (respectively, $\left.0 \neq q \in \operatorname{Spec}\left(k\left[x^{-1}\right]\right)\right)[\mathbf{1 2}, \S 54(\mathrm{I})]$. From the arguments of the proof of Proposition 4.6 and since $S^{-1} C_{1} \cong C \cong T^{-1} C_{2}$, it follows that we may restrict to primes of $\operatorname{Spec}(k[x])$ whenever $p \neq(x)$, so

$$
\begin{aligned}
C(\mathcal{M}) \equiv & \bigoplus_{(x) \neq p \in \operatorname{Spec}(k[x])} D\left(E(k[x] / p)^{\left(X_{p}\right)}\right) \oplus D\left(E(k[x] /(x))^{(S)}\right) \\
& \oplus D^{\prime}\left(E\left(k\left[x^{-1}\right] /\left(x^{-1}\right)\right)^{(Z)}\right) \oplus D\left(\prod_{(x) \neq p \in \operatorname{Spec}(k[x])} L_{p}\right) \oplus\left(L_{(x)} \rightarrow W \leftarrow U_{\left(x^{-1}\right)}\right),
\end{aligned}
$$

with $L_{(x)}, U_{\left(x^{-1}\right)}$ reduced and cotorsion $k[x]_{(x)}$ and $k[x]_{\left(x^{-1}\right)}$ modules, respectively, but this representation must be 0 because its flat cover (torsion-free cover) is equal to 0 by Lemma 5.1. Therefore, we get an expression for $C(\mathcal{M})$ in terms of the functors $D$ and $D^{\prime}$ (observe that both functors $D$ and $D^{\prime}$ preserve direct sums), which, by Lemma 4.1, allows us to compute the torsion-free cover $\varphi: \mathcal{G} \rightarrow C(\mathcal{N})$ in terms of modules (by using $[6, \S 3])$. So

$$
\mathcal{G} \equiv D\left(J^{-1} \prod_{(x) \neq p \in \operatorname{Spec}(k[x])} V_{p}\right) \oplus D\left(J^{-1} V_{(x)}\right) \oplus D^{\prime}\left(H^{-1} U_{\left(x^{-1}\right)}\right) \oplus \prod_{(x) \neq p \in \operatorname{Spec}(k[x])} D\left(U_{p}\right)
$$

(where $J=k[x]-\{0\}, H=k\left[x^{-1}\right]-\{0\}$ ) and its kernel $\mathcal{K}=\operatorname{ker}(\varphi)$ is

$$
\mathcal{K} \equiv D\left(\prod_{(x) \neq p \in \operatorname{Spec}(k[x])} V_{p}\right) \oplus D\left(V_{(x)}\right) \oplus D^{\prime}\left(U_{\left(x^{-1}\right)}\right) \oplus \prod_{(x) \neq p \in \operatorname{Spec}(k[x])} D\left(B_{p}\right),
$$

where $V_{p}, U_{p}$ and $B_{p}$ are torsion-free and cotorsion $k[x]_{p}$-modules, $0 \neq p \in \operatorname{Spec}(k[x])$ and $U_{\left(x^{-1}\right)}$ is a torsion-free and cotorsion $k[x]_{\left(x^{-1}\right)}$-module. 
To compute the coGalois group of $\varphi: \mathcal{G} \rightarrow C(\mathcal{M})$ we use the group isomorphism

$$
\operatorname{coGal}(\varphi) \cong \operatorname{Hom}_{Q \operatorname{co}\left(\boldsymbol{P}^{1}(k)\right)}(\mathcal{G}, \mathcal{K}) .
$$

Let us compute it for the first direct summand of $\mathcal{G}$ with each summand of $\mathcal{K}$ :

(i) $\operatorname{Hom}_{Q \operatorname{co}\left(\boldsymbol{P}^{1}(k)\right)}\left(D\left(J^{-1} \prod_{(x) \neq p \in \operatorname{Spec}(k[x])} V_{p}\right), D\left(\prod_{(x) \neq p \in \operatorname{Spec}(k[x])} V_{p}\right) \oplus D\left(V_{(x)}\right)\right)$

$$
\cong \operatorname{Hom}_{k[x]}\left(J^{-1} \prod_{(x) \neq p \in \operatorname{Spec}(k[x])} V_{p}, \prod_{(x) \neq p \in \operatorname{Spec}(k[x])} V_{p} \oplus V_{(x)}\right)=0,
$$

because

$$
J^{-1} \prod_{(x) \neq p \in \operatorname{Spec}(k[x])} V_{p}
$$

is a divisible $k[x]$-module and

$$
\prod_{(x) \neq p \in \operatorname{Spec}(k[x])} V_{p} \oplus V_{(x)}
$$

is a reduced $k[x]$-module;

(ii) $\operatorname{Hom}_{Q c o\left(\boldsymbol{P}^{1}(k)\right)}\left(D\left(J^{-1} \prod_{(x) \neq p \in \operatorname{Spec}(k[x])} V_{p}\right), D^{\prime}\left(U_{\left(x^{-1}\right)}\right)\right)$

because

$$
\cong \operatorname{Hom}_{k\left[x^{-1}\right]}\left(J^{-1} \prod_{(x) \neq p \in \operatorname{Spec}(k[x])} V_{p}, U_{\left(x^{-1}\right)}\right)=0
$$

$$
J^{-1} \prod_{(x) \neq p \in \operatorname{Spec}(k[x])} V_{p}
$$

is a divisible $k\left[x^{-1}\right]$-module and $U_{\left(x^{-1}\right)}$ is a reduced $k\left[x^{-1}\right]$-module;

(iii) $\operatorname{Hom}_{Q \operatorname{co}\left(\boldsymbol{P}^{1}(k)\right)}\left(D\left(J^{-1} \prod_{(x) \neq p \in \operatorname{Spec}(k[x])} V_{p}\right), \prod_{(x) \neq p \in \operatorname{Spec}(k[x])} D\left(B_{p}\right)\right)$

$$
\cong \operatorname{Hom}_{k[x]}\left(J^{-1} \prod_{(x) \neq p \in \operatorname{Spec}(k[x])} V_{p}, \prod_{(x) \neq p \in \operatorname{Spec}(k[x])} B_{p}\right)=0
$$

because

$$
J^{-1} \prod_{(x)} \neq p \in \operatorname{Spec}(k[x]) V_{p}
$$

is a divisible $k[x]$-module and

$$
\prod_{(x) \neq p \in \operatorname{Spec}(k[x])} B_{p}
$$

is a reduced $k[x]$-module. 
Similar arguments show that the only non-zero summands in the previous decomposition are

$$
\begin{aligned}
\operatorname{Hom}_{Q c o\left(\boldsymbol{P}^{1}(k)\right)}(\mathcal{G}, \mathcal{K}) \cong \operatorname{Hom}_{k[x]}( & \left.\prod_{(x) \neq p \in \operatorname{Spec}(k[x])} U_{p}, \prod_{(x) \neq p \in \operatorname{Spec}(k[x])} V_{p}\right) \\
& \oplus \operatorname{Hom}_{k[x]}\left(\prod_{(x) \neq p \in \operatorname{Spec}(k[x])} U_{p}, \prod_{(x) \neq p \in \operatorname{Spec}(k[x])} B_{p}\right) .
\end{aligned}
$$

Now, applying [16, Lemma 4.1.8], it follows that

$$
\operatorname{Hom}_{Q c o\left(\boldsymbol{P}^{1}(k)\right)}(\mathcal{G}, \mathcal{K}) \cong \prod_{(x) \neq p \in \operatorname{Spec}(k[x])} \operatorname{Hom}_{k[x]}\left(U_{p}, V_{p}\right) \oplus \operatorname{Hom}_{k[x]}\left(U_{p}, B_{p}\right) .
$$

Since $\operatorname{Hom}_{k[x]}\left(J^{-1} V_{p}, V_{p}\right)=0$ and $\operatorname{Hom}_{k[x]}\left(J^{-1} V_{p}, B_{p}\right)=0$, we can write this as

$$
\prod_{(x) \neq p \in \operatorname{Spec}(k[x])} \operatorname{Hom}_{k[x]}\left(J^{-1} V_{p} \oplus U_{p}, V_{p} \oplus B_{p}\right),
$$

which, in turn, is isomorphic to

$$
\prod_{(x) \neq p \in \operatorname{Spec}(k[x])} \operatorname{Hom}_{Q c o\left(P^{1}(k)\right)}\left(D\left(J^{-1} V_{p}\right) \oplus D\left(U_{p}\right), D\left(V_{p}\right) \oplus D\left(B_{p}\right)\right),
$$

that is, the corresponding group to the torsion-free cover of the quasi-coherent sheaf $D\left(E(k[x] / p)^{\left(X_{p}\right)}\right) \oplus D\left(L_{p}\right)$, with $(x) \neq p \in \operatorname{Spec}(k[x])$.

Now we prove the main result of the paper.

Theorem 5.3. If $\mathcal{M}$ is a quasi-coherent sheaf over $\boldsymbol{P}^{1}(k)$ and $C(\mathcal{N})$ is its cotorsion envelope, then the following assertions are equivalent:

(i) if $\psi: \mathcal{G} \rightarrow C(\mathcal{M})$ is a torsion-free cover, then

$$
\operatorname{coGal}(\psi) \cong \prod_{(x) \neq p \in \operatorname{Spec}(k[x])} G_{p},
$$

where $G_{p}$ is a locally compact modulo $G_{p}^{p}$ coGalois group, $p \in \operatorname{Spec}(k[x])$;

(ii) the cotorsion envelope $C(\mathcal{M})$ is a finite product of quasi-coherent sheaves of the form described in Proposition 4.6.

Moreover, if (i) (or equivalently, (ii)) holds, the coGalois group of a torsion-free cover of $\mathcal{M}$ is a product indexed by $\operatorname{Spec}(k[x])$ of groups $H_{p}$ that are locally compact modulo $H_{p}^{p}$, $p \in \operatorname{Spec}(k[x])$.

Proof. (i) $\Rightarrow$ (ii). This is a consequence of the decomposition (5.1) in the preceding proof, applying the argument of the proof of the necessary condition of Theorem 4.5 to the different homomorphism groups indexed on each $p \in \operatorname{Spec}(k[x]), p \neq(x)$. 
(ii) $\Rightarrow$ (i). This is given by Proposition 4.6 and Theorem 5.2, noting that the functor $D$ (respectively, $D^{\prime}$ ) preserves products.

Finally, we prove the last claim. We have the push-out-pull-back square



and we consider the sequence of topological groups,

$$
1 \rightarrow \operatorname{Hom}(\operatorname{coker}(\beta), \operatorname{ker}(\varphi)) \rightarrow \operatorname{coGal}(\psi) \rightarrow \operatorname{coGal}(\varphi) \rightarrow 1 .
$$

We shall show that $\operatorname{Hom}(\operatorname{coker}(\beta), \operatorname{ker}(\varphi)) \cong \prod_{p} \operatorname{Hom}\left(M_{p}, N_{p}\right)$, for certain completions $M_{p}$ and $N_{p}$ of free $k[x]_{p}$-modules with respect to the $p_{p}$-adic topology, $p \in \operatorname{Spec}(k[x])$. If so, we will have

$$
\operatorname{coGal}(\varphi) \cong \prod_{p} \frac{G_{p}}{\operatorname{Hom}\left(M_{p}, N_{p}\right)},
$$

so the previous sequence is the product of sequences

$$
1 \rightarrow \operatorname{Hom}\left(M_{p}, N_{p}\right) \rightarrow G_{p} \rightarrow B_{p} \rightarrow 1 .
$$

It is then straightforward to check that, if $G_{p}$ is locally compact modulo $G_{p}^{p}$, then the quotient $B^{p}$ will likewise be locally compact. So let us consider $\operatorname{Hom}(\operatorname{coker}(\beta), \operatorname{ker}(\varphi))$. By [16, Lemma 2.1.2], $\operatorname{coker}(\beta) \equiv G_{1} / F_{1} \rightarrow G / F \leftarrow G_{2} / F_{2}$ is torsion free, and, by [9, Lemma 5.1] and [16, Proposition 1.2.2], we conclude that $G_{1} \cong C\left(F_{1}\right) \oplus K$ (and an analogous argument for $\left.G_{2}\right)$, so $G_{1} / F_{1} \cong C\left(F_{1}\right) / F_{1} \oplus K$. But $C\left(F_{1}\right) / F_{1}$ is divisible and $K$ is torsion free and cotorsion, so is then a product of $M_{p}, p \in \operatorname{Spec}(k[x])$, and the same applies for $G_{2} / F_{2}=C\left(F_{2}\right) / F_{2} \oplus K^{\prime}$, with $K^{\prime}$ a product of $V_{q}, q \in \operatorname{Spec}\left(k\left[x^{-1}\right]\right)$ (see [4, Theorem]). Since $S^{-1} M_{p} \cong M_{p} \cong T^{-1} M_{p}$ whenever $p \neq(x)$, by Lemma 5.1 we see that the representation $M_{(x)} \rightarrow W \leftarrow V_{\left(x^{-1}\right)}$ cannot appear in the decomposition. This means that $\operatorname{coker}(\beta)$ is a direct sum of a quasi-coherent sheaf that is locally divisible with the cotorsion and torsion-free quasi-coherent sheaf $D\left(\prod_{(x) \neq p \in \operatorname{Spec}(k[x])} M_{p}\right)$. Finally, since $\operatorname{ker}(\varphi)$ is cotorsion, torsion-free and reduced, we see that [9, Theorem 4.7]

$$
\operatorname{ker}(\varphi)=D\left(\prod_{p \in \operatorname{Spec}(k[x])} N_{p}\right) \oplus D^{\prime}\left(\prod_{q \in \operatorname{Spec}\left(k\left[x^{-1}\right]\right)} N_{q}\right)
$$

with $N_{p}, N_{q}$ completions of free $k[x]_{p}$ and $k\left[x^{-1}\right]_{q}$ modules with respect to the $p_{p}$ and $q_{q}$-adic topologies, respectively:

$$
\begin{array}{r}
\operatorname{Hom}_{Q c o\left(\boldsymbol{P}^{1}(k)\right)}(\operatorname{coker}(\beta), \operatorname{ker}(\varphi)) \\
\cong \operatorname{Hom}_{Q \operatorname{co}\left(\boldsymbol{P}^{1}(k)\right)}\left(D\left(\prod_{(x) \neq p \in \operatorname{Spec}(k[x])} M_{p}\right), D\left(\prod_{p \in \operatorname{Spec}(k[x])} N_{p}\right)\right. \\
\left.\oplus D^{\prime}\left(\prod_{q \in \operatorname{Spec}\left(k\left[x^{-1}\right]\right)} N_{q}\right)\right) .
\end{array}
$$


An argument analogous to the proof of Theorem 5.2 allows us to write

$$
\operatorname{Hom}_{Q c o\left(\boldsymbol{P}^{1}(k)\right)}(\operatorname{coker}(\beta), \operatorname{ker}(\varphi)) \cong \prod_{(x) \neq p \in \operatorname{Spec}(k[x])} \operatorname{Hom}_{k[x]}\left(M_{p}, N_{p}\right)
$$

Definition 5.4. Let $\mathcal{M}$ be a quasi-coherent sheaf such that the associated coGalois group is the product of locally compact groups $G_{p}$ modulo $G_{p}^{p}, p \in \operatorname{Spec}(k[x])$. We call such a sheaf locally coGalois decomposable. If, moreover, these groups are in fact coGalois groups, we add 'by coGalois groups'.

Now we shall deduce some homological implications for quasi-coherent sheaves having the property of local compactness. To introduce these results we recall that a torsionfree and cotorsion $k[x]$-module $T_{p}, p \in \operatorname{Spec}(k[x])$ is defined to be of finite rank if the dimension of the $k[x] / p$-vector space $T_{p} / p T_{p}$ is finite. More generally, by a cotorsion $k[x]$-module of finite rank we mean a $k[x]$-module as in Theorem 4.5 (and the analogous definition for quasi-coherent sheaves made from the functor $D$ ). From Theorem 5.3 we immediately get the following corollary.

Corollary 5.5. Let $\mathcal{M}$ be a quasi-coherent sheaf over $\boldsymbol{P}^{1}(k)$. Then the coGalois group of $C(\mathcal{M})$ is locally coGalois decomposable by coGalois groups if, and only if, $C(\mathcal{M})$ has a minimal flat resolution $0 \rightarrow \mathcal{F}_{1} \rightarrow \mathcal{F}_{0} \rightarrow C(\mathcal{M}) \rightarrow 0$ such that the rank of each component in $\mathcal{F}_{0}, \mathcal{F}_{1}$ is finite.

Corollary 5.6. The coGalois group of a quasi-coherent sheaf $\mathcal{M}$ of $\boldsymbol{P}^{1}(k)$ is locally coGalois decomposable by coGalois groups if, and only if, $\mathcal{M}$ has a minimal cotorsion resolution $0 \rightarrow \mathcal{M} \rightarrow \mathcal{C}_{0} \rightarrow \mathcal{C}_{1} \rightarrow 0$ such that the rank of each component in $\mathfrak{C}_{0}$ and $\mathcal{C}_{1}$ is finite.

\section{Proof.}

Case $1(\Rightarrow)$. Suppose that the rank of $\mathcal{C}_{0}$ is not finite. Following the argument of the proof of Theorem 5.2, and by [9, Corollary 3.4],

$$
\begin{aligned}
& \mathcal{C}_{0} \equiv \bigoplus_{(x) \neq p \in \operatorname{Spec}(k[x])} D\left(E(k[x] / p)^{\left(X_{p}\right)}\right) \oplus D\left(E(k[x] /(x))^{(S)}\right) \\
& \oplus D^{\prime}\left(E\left(k\left[x^{-1}\right] /\left(x^{-1}\right)\right)^{(Z)}\right) \oplus \prod_{(x) \neq p \in \operatorname{Spec}(k[x])} D\left(L_{p}\right),
\end{aligned}
$$

with some $X_{p}$ (or $S$ or $Z$ ) infinite, $p \in \operatorname{Spec}(k[x]$ ). But then the coGalois group associated with $\mathcal{M}$ cannot have the property of Theorem 5.3. So the rank of $\mathcal{C}_{0}$ is finite. This automatically implies that the rank of $\mathcal{C}_{1}$ is also finite.

Case $2(\Leftarrow)$. If $\mathcal{C}_{0}$ is of finite rank, it follows that it is of the form of Proposition 4.6, so this result, together with Theorem 5.3, means that the coGalois group is as desired. 
Now we will see that locally coGalois decomposable quasi-coherent sheaves are generalizations of finitely generated and finitely cogenerated quasi-coherent sheaves.

Corollary 5.7. Every finitely generated quasi-coherent sheaf on $\boldsymbol{P}^{1}(k)$ is locally coGalois decomposable.

Proof. Let $\mathcal{M} \equiv M_{1} \rightarrow P \leftarrow M_{2}$ be a finitely generated quasi-coherent sheaf on $\boldsymbol{P}^{1}(k)$. Then there exists an epimorphism $\bigoplus_{i=1}^{t} \mathcal{O}\left(n_{i}\right) \rightarrow \mathcal{M} \rightarrow 0$ (where $\bigoplus_{i=1}^{t} \mathcal{O}\left(n_{i}\right)$ is a finite direct sum of twists of the structure sheaf). So $M_{1}$ (and $\left.P, M_{2}\right)$ is a finitely generated module of the form

$$
M_{1}=k[x] / p_{1}^{r_{1}} \oplus k[x] / p_{2}^{r_{2}} \oplus \cdots \oplus k[x] / p_{s}^{r_{s}} \oplus k[x]^{n},
$$

and a similar kind of decomposition occurs for $M_{2}$. But, since

$$
k[x] /\left(x-u_{i}\right)^{r^{i}} \cong k\left[x^{-1}\right] /\left(x^{-1}-u_{i}^{-1}\right)^{r^{i}}
$$

whenever $u_{i} \neq 0$, it follows that

$$
\mathcal{M} \equiv D\left(k[x] / p_{1}^{r_{1}}\right) \oplus D\left(k[x] / p_{2}^{r_{2}}\right) \oplus \cdots \oplus D\left(k[x] / p_{s}^{r_{s}}\right) \oplus k[x]^{n} \rightarrow k\left[x^{-1}, x\right]^{n} \leftarrow k\left[x^{-1}\right]^{n} .
$$

Now the cotorsion envelope of $k[x]^{n} \rightarrow k\left[x^{-1}, x\right]^{n} \leftarrow k\left[x^{-1}\right]^{n}$ is

$$
D\left(\prod_{u} \widehat{k[x]}_{(x-u)}^{n}\right) \oplus D^{\prime}\left({\widehat{k\left[x^{-1}\right]}}_{\left(x^{-1}\right)}^{n}\right)
$$

(this is a consequence of [9, Corollary 3.4] and [16, Corollary 4.2.4]), so it is of the form of Proposition 4.6.

As a consequence of Corollary 5.7 we supply a wide family of quasi-coherent sheaves with the property of Theorem 5.3.

Corollary 5.8. Every vector bundle of $\boldsymbol{P}^{1}(k)$ is locally coGalois decomposable.

Locally coGalois decomposable quasi-coherent sheaves also generalize finitely cogenerated sheaves of $Q \operatorname{co}\left(\boldsymbol{P}^{1}(k)\right)$.

Corollary 5.9. Let $\mathcal{M}$ be a finitely cogenerated quasi-coherent sheaf of $\boldsymbol{P}^{1}(k)$. Then the coGalois group associated with $\mathcal{M}$ has the property of Theorem 5.3(i).

Proof. First of all, if $\mathcal{M}$ is finitely cogenerated it is locally finitely cogenerated, because injective envelopes are computed componentwise. But a finitely cogenerated $k[x]$-module $M_{1}$ is a finite direct sum of cocyclic $k[x]$-submodules, that is, of the form $k[x] / p^{r}$ and $E(k[x] / p)\left[\mathbf{1 2}\right.$, Theorem 25.1]. So then $\mathcal{M} \equiv M_{1} \rightarrow P \leftarrow M_{2}$ is a cotorsion module of the form of Proposition 4.6, and therefore the corresponding coGalois group decomposes as the product of locally compact groups modulo $G_{p}^{p}, p \in \operatorname{Spec}(k[x])$.

Acknowledgements. The authors are partly supported by the DGI MTM200503227. The final version of this paper was completed during S.E.'s stay at the Department of Mathematics of the University of Kentucky with the support of an MEC/Fulbright Grant from the Spanish Secretaría de Estado de Universidades e Investigación del Ministerio de Educación y Ciencia.

The authors thank the referee for many helpful comments and suggestions. 


\section{References}

1. P. BAUMANN AND C. KASSEL, The Hall algebra of the category of coherent sheaves on the projective line, J. Reine Angew. Math. 533 (2001), 207-233.

2. E. Enochs, Torsion free covering modules, II, Arch. Math. 22 (1971), 37-52.

3. E. ENOCHS, Injective and flat covers, envelopes and resolvents, Israel J. Math. 39 (1981), 189-209.

4. E. Enochs, Flat covers and flat cotorsion modules, Proc. Am. Math. Soc. 92 (1984), 179-184.

5. E. ENOChS AND S. EstradA, Relative homological algebra in the category of quasicoherent sheaves, Adv. Math. 194 (2005), 284-295.

6. E. Enochs And S. Estrada, The structure of compact coGalois groups, Houston J. Math. 32 (2006), 961-970.

7. E. Enochs, J. R. García Rozas, O. M. G. Jenda and L. Oyonarte, Compact coGalois groups, Math. Proc. Camb. Phil. Soc. 128 (2000), 233-244.

8. E. Enochs, S. Estrada, J. R. García Rozas and L. Oyonarte, Generalized quasicoherent sheaves, J. Alg. Appl. 2 (2003), 63-83.

9. E. Enochs, S. Estrada, J. R. García Rozas and L. Oyonarte, Flat and cotorsion quasi-coherent sheaves: applications, Alg. Representat. Theory 7 (2004), 441-456.

10. E. Enochs, S. Estrada And J. R. García-Rozas, Galois and coGalois groups associated with cotorsion theories, Houston J. Math. 32 (2006), 651-663.

11. S. EstradA, Cubiertas y envolventes en categorías de representaciones, PhD thesis, University of Almería (2003).

12. L. FUCHS, Infinite abelian groups, Volume I (Academic, 1970).

13. N. KoBLITZ, $p$-adic numbers, $p$-adic analysis and zeta-functions, Graduate Texts in Mathematics, Volume 58 (Springer, 1977).

14. L. SAlCE, Cotorsion theories for abelian groups, Symp. Math. 23 (1979), 11-32.

15. H. H. SchaEfER, Topological vector spaces, Graduate Texts in Mathematics, Volume 3 (Springer, 1971).

16. J. XU, Flat covers of modules, Lecture Notes in Mathematics, Volume 1634 (Springer, 1996). 